Objectives: To objectively evaluate the RA hand's speed of the fine movements, through the HTS and to compared with a group of age and sex matched healthy controls. To verify the correspondence with the HAQ, DAS28, GS

Methods: 55 consecutives RA patients (pts) ( 6 males, age $61 \pm 16$ years, mean duration of disease $12 \pm 8$ years), classified according to 2010 ACR/EULAR criteria6, and 50 matched healthy controls (HCs) were enrolled. After consent, all participants undergone HTS test that recognizes the touches between the finger tips during the opposition movements of the hands in standard sequences of movements, after dressed the glove. A multiple finger evaluation (MFE) and a single finger evaluation (SFE) were performed using a dedicated software that provided the physician the following quantitative parameters: Touch Duration (TD), Inter Tapping Interval (ITI) and Movement Rate (MR). Average time for hand 2 minutes. RA pts compiled the HAQ, performed the GS and a DAS28cpr was performed.

The student's t-test was used to compare the glove's parameters between the groups whereas the analysis of variance (ANOVA) was utilized to verify potential differences between the populations. In order to evaluate the single correlations, the $r$ and $p$ values of Pearson were employed.

Results: For MFE, glove parameters TD and ITI were significantly higher in RA pts than HCs, whereas; MR was significantly lower in RA pts compared to HCs (all $p<0.001$ ).

For SFE non-affected fingers (not swollen and not tender) of RA pts performed better than a clinically affected fingers, but in any case significantly worse than average HCs fingers $(p<0.001)$.

There is a statistically significant correlation between the GS and MR $(r=0.39$ $\mathrm{p}=0.003)$ and TD $(\mathrm{r}=-0.33 \mathrm{p}=0.015)$.

TD, ITI e MR of RA pts showed a significant correlation with the total score of the HAQ ( $r=0.56, r=0.39, r=-0.56$, all $p<0.001 ;)$. DAS28, considered as a continuous variable, proved to be significantly correlated with the TD $(r=0.36, p$ $=0.009)$. When the RA patients were grouped according to the disease activity by DAS28cpr7, there was an increase of one third of the TD's logarithm for each increase in the activity class (linear regression with ordinal predictors, beta = $0.33 ; 95 \% \mathrm{Cl} 0.03,0.63, \mathrm{p}<0.0297)$. Finally, even RA pts in remission showed a TD significantly higher compared with $\mathrm{HCs}(p=0.034)$.

Conclusion: The RAGLOVE is shown as a new safe and fast tool to evaluate a new objective parameter in the hand's functionality: the speed of finger movements. In RA pts, an inversely proportional correlation emerges between the speed of movement and disease activity.

The significant correlation found with $\mathrm{HAQ}$, highlights the loss of motility of the hands as one of the main determinant of disability. The RAGLOVE is now tested in RA patients undergoing treatment.

REFERENCES:

[1] Hakkinen et al Ann Rheum Dis. 2005;

[2] Van Der Heijde et al J of Rheum. 1993;

[3] Fries et al Arthritis Rheum. 1980;

[4] Mathiowetz et al J Hand Surg Am. 1984;

[5] Carmisciano et al Eur J Neurol. 2020;

[6] Aletaha et al. Ann Rheum Dis. 2010;

[7] Aletaha et al Arthritis Rheum 2005.

Disclosure of Interests: None declared

DOI: 10.1136/annrheumdis-2021-eular.3991

\section{POS0584 ULTRASONOGRAPHY OF THE MEDIAN NERVE IN PATIENTS WITH RHEUMATOID ARTHRITIS UNDER SUSPICION OF CARPAL TUNNEL SYNDROME}

S. Tsiami ${ }^{1}$, E. Ntasiou ${ }^{2}$, C. Krogias ${ }^{2}$, R. Gold ${ }^{2}$, J. Braun ${ }^{1}$, M. Sarholz ${ }^{3}$, X. Baraliakos ${ }^{1} .{ }^{1}$ Ruhr-University Bochum, Rheumazentrum Ruhrgebiet, Herne, Germany; ${ }^{2}$ Ruhr-University Bochum, St. Josef-Hospital, Bochum, Germany; ${ }^{3}$ St. Marien-Hospital Vreden, Klinik fuer Rheumatologie, Vreden, Germany

Background: Carpal tunnel syndrome (CTS) is the most common nerve compression syndrome and a common extra-articular manifestation of rheumatoid arthritis (RA). Different causes of CTS are known, among them inflammatory and non-inflammatory pathologies. Electroneurography (ENG) of the median nerve, the method of choice to diagnose CTS, measures impairment of nerve conduction velocity without explaining its underlying cause. However, because the electrical stimulation is often not well tolerated, ENG results may come out inconclusive. Using greyscale ultrasonography (GS-US) provides anatomic information including a structural representation of the carpal tunnel.

Objectives: To investigate the performance of nerve GS-US in the diagnosis of CTS in patients with RA.

Methods: Consecutive patients with active RA under suspicion of CTS presenting to a large rheumatologic center were included. Both hands were examined by an experienced neurologist including ENG and a GS-US (ML linear probe with 6-15 Hz) of the median nerve. An established grading system for ENG (1), and an established system for GS-US based on cut-offs for the nerve cross sectional area (CSA) [mild: $0,11-0,13 \mathrm{~cm}^{2}$, moderate: $0,14-0,15 \mathrm{~cm}^{2}$, severe: $>0,15 \mathrm{~cm}^{2}$ CTS
(2)] were used. In addition, the Boston Carpal Tunnel Syndrome Questionnaire (BCTSQ) was used to assess CTS symptoms (3).

Results: Both hands of 58 patients with active RA $(n=116)$ and clinical suspicion of CTS (in 38 cases bilaterally) were included. After clinical examination, CTS was suspicious in 96 hands (82.8\%), and 59 of all hands had a final diagnosis of CTS $(50.9 \%)$. Of the latter, 43 hands $(72.9 \%)$ had a positive ENG and 16 $(27.1 \%)$ a positive GS-US finding only, while 30 hands $(50.8 \%)$ were positive in both examinations.

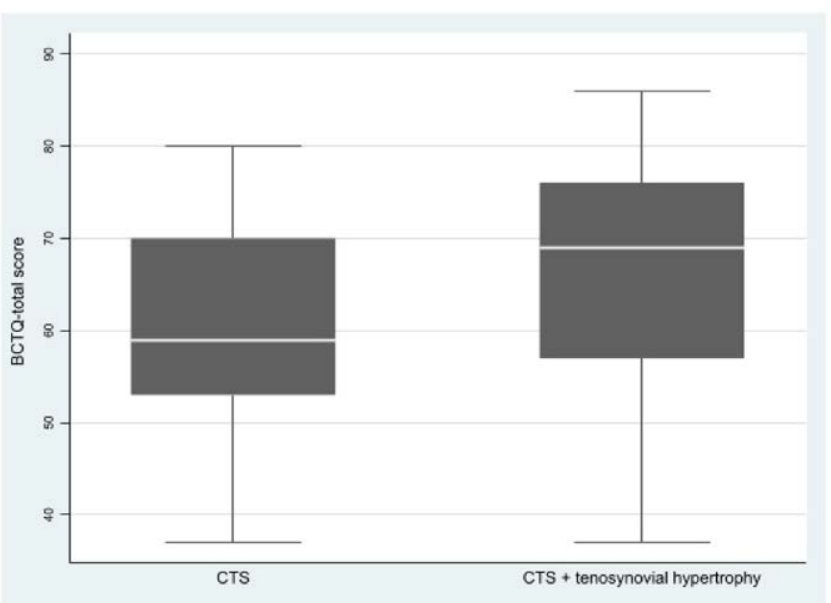

Figure 1. BCTSQ scores in patients with diagnosis of CTS and absence or presence of RA-related tenosynovial hypertrophy

There was a good correlation of the cross-sectional area (CSA) as well as the CSA-ratio to the ENG findings: the larger the CSA, the more severe was the CTS as assessed by ENG (Spearman's rho $=0.554$; $p<0.001$ ). The more severe the GS-US findings of CTS were, the more definite were the distal motor latency (Spearman's rho $=0.554 ; p<0.001$ ) and sensible nerve conduction velocity of the median nerve (Spearman's rho $=-0.5411 ; p<0.001$ ).

In the 46 hands positive in GS-US, tenosynovial hypertrophy of the flexor tendons was detected in 19 hands (41.3\%), 7 of which (36.8\%) also showed an additional cystic mass. In these 19 patients, clinical complains were more severely present than in patients with non-inflammatory CTS, as assessed by the BCTSQ with a total score of $68.8 \pm 13.4$ vs. $59.3 \pm 13.7$, respectively $(p=0.007)$.

Conclusion: In patients with active RA and clinical complains of CTS, ultrasound examinations provide additional information about inflammation which is helpful for a diagnosis of CTS. Thus, ENG and nerve GS-US should be used complementary for a diagnostic workup of CTS in RA patients with a suspicion of CTS. Power-Doppler may further improve the diagnostic performance of GS-US. REFERENCES:

[1] Padua L et al. Acta Neurol Scand 1997; 96:211-217

[2] El Miedany et al., Rheumatology (Oxford). 2004 Jul; 43(7):887-895

[3] Levine DW et al. J Bone Joint Surg Am 1993; 75: 1585-1592

Disclosure of Interests: None declared

DOI: 10.1136/annrheumdis-2021-eular.3994

\section{ORAL STATUS IN PATIENTS WITH RHEUMATOID ARTHRITIS}

A. Ben Salem ${ }^{1}$, M. Brahem ${ }^{1}$, H. Hachfi ${ }^{1}$, R. Sarraj ${ }^{1}$, S. Abdellatif ${ }^{1}$, R. Ben Tekaya ${ }^{1}$, C. Baccouche ${ }^{2}$, Y. Mohamed ${ }^{1} .{ }^{1}$ Tahar Sfar Hospital, Rheumatology, Mahdia, Tunisia; ${ }^{2}$ Tahar Sfar Hospital, Dentistry, Mahdia, Tunisia

Background: Rheumatoid arthritis (RA) is a systemic auto immune inflammatory disorder, which primarily affects the synovial joints, but it may have an impact on oral health.

Objectives: The present study aimed to describe oral manifestations in patients with Rheumatoid arthritis (RA)

Methods: A cross sectional study including RApatients, which consulted or were hospitalized in Rheumatology department in Taher Sfar Hospital of Mahdia Tunisia, during a period of 10 months. The diagnosis of RA was based on ACR/EULAR 2010 criteria. Oral and periodontal examination was practiced by a trained dentist in the same hospital. Oral hygiene, teeth status (missing teeth) and paradental parameters (bleeding index of Loe and Silness (IG), plaque indexed O'Leary (IP) were determined for each patient, to assess oral and periodontal diseases in RA.

Results: Our study included 51 patients with an average age of 51.11 years \pm 12.4 [21-74years].50patients (92.6\%of cases) were women and 8patients (14.8\%) were diabetic. Only one patient was a smoker. The mean duration of RA was 10.7 years $7.7 \pm$ [10months-35years]. Rheumatoid factor (RF) was positive 